\title{
Nutrition and quality of life in chronic kidney disease patients: a practical approach for salt restriction
}

\author{
Kunitoshi Iseki ${ }^{1,2,3}$ \\ ${ }^{1}$ Clinical Research Support Center, Nakamura Clinic, Okinawa, Japan \\ ${ }^{2}$ Okinawa Dialysis and Transplant Association, Okinawa, Japan \\ ${ }^{3}$ Okinawa Heart and Renal Association, Okinawa, Japan
}

The clinical practice guidelines (CPGs) for nutrition in chronic kidney disease (CKD) were updated after 20 years from the previous guidelines by the Kidney Disease Outcomes Quality Initiative (KDOQI). During this period, the severity of CKD was defined by eGFR and albuminuria by the organization Kidney Disease: Improving Global Outcomes (KDIGO). Main risk factors for CKD such as hypertension, hyperlipidemia, obesity, metabolic syndrome, and diabetes mellitus are closely related to lifestyle. Nutritional management is important to prevent and retard the progression of CKD. Members of the International Society of Renal Nutrition and Metabolism (ISRNM) reviewed the KDOQI CPG draft. ISRNM is an international scientific society comprising members of multiple subspecialties. ISRNM proposed the medical term protein-energy wasting (PEW), which is a keyword in renal nutrition. The prevalence of PEW among dialysis patients is high. The success of dietary therapy depends on adherence to the diet. It has to be palatable, otherwise eating habits will not change. To prevent the development and progression of CKD and PEW, regular consultation with an expert dietitian is required, especially regarding salt and protein restriction. Our cluster-randomized trial showed that intervention by a dietician was effective at retarding the progression of stage 3 CKD. In this review, I focus on salt (sodium) restriction and introduce tips for salt restriction and Japanese kidney-friendly recipes. Due to the lack of randomized controlled trials, nutritional management of CKD inevitably relies on expert opinion. In this regard, well-designed observational studies are needed. Too strict salt restriction may decrease quality of life and result in PEW.

Keywords: Chronic kidney disease, Dialysis, Hypertension, Salts, Sodium

\section{Introduction}

Chronic kidney disease (CKD) is increasing worldwide and is common in the elderly population [1]. It is commonly associated with lifestyle-related conditions such as diabetes mellitus (DM), hypertension, hyperlipidemia, smoking, and obesity. Information regarding lifestyle factors such as smoking, drinking alcohol, exercise, sleep, and eating habits is usually obtained during screening and daily medical practice. Correction of an unhealthy lifestyle often has favorable outcomes. For instance, recovery from metabolic syndrome was associated with a reduced risk of CKD [2]. In addition to pharmacological management, nutritional management is an important strategy in CKD patient care.

Received: September 11, 2021; Revised: October 24, 2021; Accepted: October 25, 2021

Correspondence: Kunitoshi Iseki

Clinical Research Support Center, Nakamura Clinic, 4 Chome-2-1 Iso, Urasoe, Okinawa 901-2132, Japan. E-mail: chihokun_ohra@yahoo.co.jp ORCID: https://orcid.org/0000-0001-6624-0216 
The Kidney Disease Outcomes Quality Initiative (KDOQI) clinical guidelines for nutrition in CKD [3] were updated after 20 years from the previous guidelines in 2000 [4]. Severity of CKD was determined using the "heat map" published in 2013 by Kidney Disease: Improving Global Outcomes (KDIGO) [5]. The first draft was finished by 2016 with an extensive review of related references. However, during the review process with stakeholders, there were a lot of questions, comments, and discussion and the final draft was only published four years later in 2020. In Japan, two major scientific associations are involved in CKD patient care: the Japanese Society of Nephrology (JSN) and the Japanese Society for Dialysis Therapy (JSDT). Both these associations work on early detection and prevention of the progression of CKD to end-stage kidney disease (ESKD), which requires dialysis and renal transplantation [6-8].

Salt restriction and protein restriction are two major aspects of nutritional management in CKD patients. However, dietary habits differ by culture, ethnicity, and other socioeconomic conditions as well as individually. Physicians taking care of CKD patients often do not have enough experience and knowledge regarding dietary management. Twenty-four-hour urine sodium excretion does not reflect actual intake as CKD progresses [9]. In chronic hemodialysis (HD) patients, failure of salt (and potassium) restriction is fatal. Therefore, collaboration with dieticians is needed during all stages of CKD. However, there is a paucity of strong evidence based on randomized controlled trials (RCTs) for dietary management in CKD patients, reflecting difficulty in recruiting sufficient patients and the required duration of interventions. Practical approaches may vary with country or district, cultures, stages of CKD, and socioeconomic conditions, but may provide useful knowledge for others concerning nutritional management.

Clinical manifestations of excess salt intake in CKD patients are hypertension, edema, and proteinuria. Regular checks of blood pressure, body weight, and urine tests can help detect CKD at an early stage. Follow-up intervals at the clinic or hospital vary with stage of CKD from every 2 weeks to annually. In this regard, self-check of blood pressure and body weight at home can be helpful. Information technology may help facilitate communication between CKD patients and physicians remotely. This is reflected by the clinical practice point added in the recent KDIGO guidelines of "Think Globally and Act Locally."

\section{Guidelines and guidance}

The KDOQI guidelines recommend limiting sodium intake to less than $100 \mathrm{mmol} /$ day (or $<2.3 \mathrm{~g} /$ day) sodium or $<5.8 \mathrm{~g}$ salt to reduce blood pressure and improve volume control in CKD 3 to 5, CKD 5D, or posttransplantation patients. Moreover, in adults with CKD 3 to 5D, restriction of dietary sodium intake has been proposed as an adjunctive lifestyle modification strategy to achieve better volume control and more desirable body weight. Recently, the KDIGO clinical practice guidelines for the management of hypertension in CKD were published [10]. In the top 10 takeaways for clinicians, sodium restriction was ranked number 7 . Low sodium ( $<2 \mathrm{~g} /$ day) or salt ( $<5 \mathrm{~g} /$ day) intake and moderate-intensity physical activity ( $\geq 150 \mathrm{~min} /$ week) were recommended for CKD patients with high blood pressure.

KDOQI guidelines were summarized in the International Society of Renal Nutrition and Metabolism (ISRNM) journal [11]. Effective medical nutritional therapy in CKD requires shared goals by all stakeholders including dietitians and other healthcare professionals. Well-designed observational studies and RCTs with nutritional and dietary interventions are needed. ISRNM published the consensus statement on "Eating during Hemodialysis Treatment" in 2018 [12].

Dietary habits differ by culture, ethnicity, and other socioeconomic factors. In Japan, the JSN and JSDT have published evidence-based clinical practice guidelines and guidance for CKD patient care [6-8]. The 18th Congress of the ISRNM was held in Okinawa, Japan in April 2016. Dietary management in CKD patients was one of the main topics. After this Congress, practical guidelines for salt and protein restriction to retard the progression of CKD to ESKD were summarized with the goal of promoting further collaboration between nephrologists and dieticians [13].

\section{Practical salt restriction}

Seven target interventions were identified. Cessation of smoking and maintaining normal body weight as reflected by a body mass index (BMI) of $<25 \mathrm{~kg} / \mathrm{m}^{2}$ were ranked first and second, with salt restriction third [13]. For hypertensive CKD patients, we suggested a salt intake of 3 to $6 \mathrm{~g}$ per day. Modification of lifestyle is a difficult and time-consuming process. We recommended at least 30 minutes face-to-face 
guidance sessions by a dietician, every 3 months if needed. Unless salt intake is adequately controlled, edema, heart failure, and hypertension can result. We recommended careful estimation of the daily intake of foods and additives. Salt intake may vary with cooking processes, in particular commercial processes.

For salt and protein restriction in individual patients, laboratory and medication data are required. Therefore, collaboration between physicians, nephrologists and/or general physicians, and dieticians is mandatory. After a thorough interview, we start salt and protein restriction separately. We show the participant how to roughly estimate salt content by using the amount of salt per one teaspoon. One teaspoon contains about $6 \mathrm{~g}$ of salt. A single-day dietary recall is used to semi-quantitively estimate the amount of sodium intake. Urine collection for 24 hours to measure sodium excretion is not feasible for the general population. It is difficult to evaluate the amount of sodium in processed foods. When sodium content is expressed as "mg," salt content should be calculated using the following formula: salt $(\mathrm{g})=\mathrm{Na}(\mathrm{mg}) \times 2.54 / 1,000$. One gram of sodium is equal to $43.5 \mathrm{mEq}$.

Estimating salt content in processed foods is important. A rough estimation of sodium is helpful for daily life. Dietary recall is necessary and may need help from trained interviewers. There is a wide variety of "junk foods" available for purchase. Generally, restaurant food and take-out foods are salt-rich, although this may differ by restaurant or region. Tips on how to evaluate salt content are summarized in Fig. 1. Commonly ordered foods in Japan are hamburgers, curry rice, and ramen. The salt content of hamburgers and curry rice is about $1.6 \mathrm{~g}$ and $3.4 \mathrm{~g}$, respectively. One bowl of salt ramen contains more than $10 \mathrm{~g}$ of salt. However, salt intake may differ by seasoning and quantity of soup. A list of high salt foods is provided in Fig. 2. Tips to estimate salt content are listed in Fig. 3. Reducing miso soup and pickle consumption is an effective way to reduce dietary salt intake [14]. Soy sauce is common in Japan but contains a lot of salt. Low-salt soy sauce is available, but potassium content is high. This is important for dialysis patients to be aware of. Dashi Wari soy sauce is tasty and has a low potassium content. Therefore, it is recommended for those who require potassium restriction. Fig. 4 summarizes the list of seasoning materials that can help reduce salt intake.
1) Ask for low-salt cooking at restaurants
2) Do not drink soup
3) Use separate dishes for dressing or mayonnaise
4) Select foods that are easy to estimate salt intake
5) When eating out, restrict salt more than usual at home

Figure 1. Tips for salt restriction when buying cooked foods and eating out. Modified from Iseki and Yamagata [13] according to the Creative Commons License.
1) Fast foods: burgers, fries, chicken fingers, and pizza
2) Salty snack foods: salted pretzels, chips, salted nuts, and salted crackers
3) Frozen dinners: frozen meat dishes and frozen pizza
4) Processed meats: bacon, sausage, lunch meat, and hot dogs
5) Cheese and dairy: cheese, cottage cheese, and buttermilk
6) Sauces and condiments: soy sauce, commercial tomato sauce, and salad dressing
7) Drinks: regular vegetable juice, juice blends, and salty alcoholic beverages
8) Seasonings: salt and salt blends

Figure 2. List of high salt foods. Modified from Iseki and Yamagata [13] according to the Creative Commons License.
1) Use a spoon to estimate the amount of added seasoning, in particular, when using common seasoning materials such as table salt, source, and miso
2) Estimate roughly as salt (grams) per teaspoon
3) Select low-salt seasoning and law salt foodstuffs (avoid too much)
4) Check the amount of salt in each food item

Figure 3. Tips to estimate salt content. Modified from Iseki and Yamagata [13] according to the Creative Commons License. 
1) Acid: vinegar, squeeze of lemon, citrus sudachi, citron, etc

2) Condiments: ginger, garlic, leaf of the perilla, leeks, etc.

3) Umami (taste): soup stock made using bonito, konbu (dried kelp), shiitake, chicken broth, small dried sardines, etc.

4) Spices: wasabi mustard (powder), curry powder, pepper, red pepper, Japanese pepper, chili oil, basil, thyme, nutmeg, paprika, cinnamon, onion powder, common sage, all-spice, etc.

Figure 4. List of seasoning materials to reduce salt intake. Modified from Iseki and Yamagata [13] according to the Creative Commons License.

Japanese Kidney-Friendly Recipes and tips on salt restriction are available in the homepage of ISRNM (Patients' Corner). Dashi (broth) and soup recipes are provided as are tips on how to reduce salt in instant noodles. The top three salted foods consumed in Japan are instant ramen, salted plums, and pickles. Instant ramen has been on the market in Japan since August 1958. It is cheap and easy to cook, but very salty. Dashi (broth) is a tasty low-sodium meal. Umami mushrooms and vegetables (especially root vegetables) are added, and spices and herbs are utilized. The YouTube site advises checking water hardness and recommends using soft water with less $\mathrm{Ca}$ and $\mathrm{Mg}$. If needed, a water softener can be used. Use of soft water can reduce 'nigami' (bitter taste) and 'egumi' (harshness). Three keys to reducing salt in stews are 1) to combine dashi with poultry and mushrooms, 2) to add soy sauce at the end, and 3) to leave out the cooking liquid to reduce the salt even further. When preparing simmered vegetables and chicken, it is important to stick to the order of adding seasonings with sugar first and salt second. In 1971, cup noodles were invented. They are cheap and easy to cook, requiring only the addition of hot water. To reduce salt but preserve taste, remove the hot water after 1 minute and then add hot water again and wait 2 minutes, and eat it. This method can reduce the amount of salt by $50 \%$.

\section{General population}

In Japan, the leading cause of ESKD since 1998 has been DM with fewer ESKD cases due to chronic glomerulonephritis. The main causes of DM are obesity [15] and metabolic syndrome. Diagnostic criteria for metabolic syndrome in Asians are different from in Western populations [16]. The cutoff waist circumference values measured at the umbilicus are $85 \mathrm{~cm}$ for men and $90 \mathrm{~cm}$ for women [17]. Metabolic syndrome is common and a predictor of both the incidence and progression of CKD in the general population [18-21].

Higher sodium intake is associated with incident CKD $[22,23]$. Recent meta-analysis supported that higher salt intake increased the odds of developing CKD [24]. Our group has been using the nationwide screening program of the 'Specific Health Check-up and Guidance System (Tokutei-Kensin)' initiated in $2008[25,26]$. The main purpose of this program is to detect metabolic syndrome and promote lifestyle modifications for people aged 40 to 74 years. In Japan, dipstick proteinuria, but not albuminuria, is used for general health screenings. Measurement of microalbuminuria is only reimbursed for early-stage diabetic kidney disease. We have shown that dipstick proteinuria is an independent predictor of death among screening participants [27]. We also found by analysis of a self-administered questionnaire on medical history and lifestyle that a 1-year change toward a healthy lifestyle was associated with a decline in proteinuria [28]. Moreover, we found that consuming fast foods is a risk factor for incident DM [29].

The effect of sodium intake on the development of CKD in a prospective cohort of people with normal renal function was investigated [30]. Sodium intake was estimated using a 24-hour dietary recall Food Frequency Questionnaire. Both individuals with low sodium intake $(<2.08 \mathrm{~g} /$ day [5.28 g salt]) and those with high sodium intake ( $>4.03$ $\mathrm{g} /$ day sodium [10.2 $\mathrm{g}$ salt]) were at higher risk of developing CKD in the presence of hypertension $(n=3,106)$ but not in the absence of hypertension $(n=4,871)$. In other words, the incidence of CKD was different between hypertensive and non-hypertensives. Adequate sodium intake appears to be important in individuals with hypertension as both too little and too much salt appear to be hazardous. Excess sodium restriction may activate the renin-angiotensin-aldosterone system, sympathetic nervous system, and aggravate insulin resistance [31]. 
The importance of lifestyle modification is not yet fully appreciated, at least among general physicians [32,33]. Other unhealthy lifestyle factors such as disturbed sleep, a sedentary lifestyle, and constipation should be taken into consideration when treating CKD patients. The prevalence of obstructive sleep apnea is high among Japanese non-dialysis CKD patients [34,35]. Nocturnal hypoxemia is a risk factor for rapid decline of kidney function [36]. We showed that treatment of sleep apnea syndrome by continuous positive airway pressure was life-saving [37]. CKD is often asymptomatic, therefore late referral to a nephrologist is not uncommon [38-40]. Nausea and vomiting are often the first presentations of CKD patients. CKD is associated with the presence of dysbiosis, which can be defined as an "imbalanced intestinal microbial community with quantitative and qualitative changes in the composition and metabolic activities of the gut microbiota" $[41,42]$. Constipation is common and related to CKD severity [43].

\section{Stage 3 to 5 chronic kidney disease patients}

Several studies have shown the beneficial effects of an educational program in CKD patients [44-46]. However, more collaboration with general physicians is needed. After the publication of the KDIGO guidelines [5], the term "CKD" has become more popular among nonspecialists, mass media, and laypeople. We care for CKD patients following the KDIGO guidelines [5,10,47], in particular the Japanese version [6-8]. Following these guidelines, we focus on individuals with metabolic syndrome as good targets for nutritional intervention.

We published a cluster-randomized trial on the effect of behavior modification in early to moderate stage CKD [48]. We estimated the effects of intervention by dieticians on lifestyle modification. A total of 2,379 patients $(1,195$ in group A that received standard interventions and 1,184 in group B who received advanced interventions), aged between 40 and 74 years, had CKD and were under management by general physicians. All general physicians belonged to local medical associations. Group B patients received three additional interventions: educational intervention for lifestyle modification (including sodium and protein restriction, if needed) and a CKD status letter to prevent their withdrawal from treatment, while group B general physicians received data sheets to facilitate reduc- ing the gap between target and practice. For this intervention, we collaborated with registered dieticians of the Japan Dietic Association.

The difference in cumulative incidence of a doubling of serum creatinine or $50 \%$ reduction in estimated glomerular filtration rate (eGFR) gradually increased between groups A and B over a follow-up of up to 3.5 years. We concluded that our care system achieved behavior modification of CKD patients, namely significantly fewer discontinuous clinical visits and behavior modification of both GPs and nephrologists, namely significantly higher referral and co-treatment rates, resulting in retardation of CKD progression, especially in patients with proteinuric stage 3 CKD. Registered patients received more than 10 interviews of 30 minutes in duration every 3 months during the study. Furthermore, we are analyzing the results of 10 years of observation of the initial cohort and focused the patients with advanced CKD and eGFR of $<45 \mathrm{~mL} / \mathrm{min} / 1.73 \mathrm{~m}^{2}$ in this cohort [49]. Also, report results using a Markov model, Okubo et al. [50] evaluated the cost-effectiveness of this intervention and found that the intervention used in this study was an efficient use of finite healthcare resources in Japan based on calculated cost per quality-adjusted lifeyear.

There are five basic tastes: sweet, sour, salty, umami, and bitter. In CKD, specific impairment in sour, umami, and salty tastes has been reported [51]. Proper dietary management and a healthy lifestyle are effective at reducing the incidence and progression of CKD, and therefore the incidence of ESKD requiring dialysis. Too high and too low salt intake are both dangerous. Recently, Kang et al. [52] showed that high salt intake, more than $11.3 \mathrm{~g} /$ day, was a risk factor for CKD progression. In a study based in an Italian nephrology clinic, salt intake of $<6 \mathrm{~g} /$ day posed a greater risk of ESKD than salt intake of $\geq 6 \mathrm{~g} /$ day [53].

\section{Dialysis patients}

HD is a life-saving therapy for those with ESKD, yet the prognosis remains poor [54]. Unfortunately, no RCTs have reported survival benefits according to dialysis-related factors such as session time, frequency, or dialyzer membrane area [55]. There are also unfortunately no clinical practice guidelines for dialysis. At the initiation of dialysis therapy, various comorbid conditions such as stroke, cardiovascular 
disease, diabetic complications, and cancers may be present. Medical treatments such as erythropoietin stimulating agents, statins, and antihypertensives have failed to show survival benefits. Furthermore, well-designed RCTs have shown that pharmacological interventions offer no survival benefits [56-58].

The mortality rate of Japanese dialysis patients is lower than that of European and American dialysis patients [59] for reasons that are not yet clear. Consequently, careful rounds at each dialysis session and timely laboratory data are required by the physician in charge and other staff. Moreover, the number of elderly patients who require additional assistance with factors like transport, communication, and other personal issues is increasing. Decisions not to undergo dialysis or withdraw from dialysis are serious problems even in developed countries [60]. Meticulous control of known predictors of survival is important [61]. Also, the importance of nutritional management, in particular on PEW, is increasingly being recognized not only in dialysis patients but also in predialysis patients [62-64].

We previously reported that serum albumin (S-Alb) was a strong predictor of death among chronic dialysis patients [65]. At that time, we assumed that S-Alb functioned as a proxy of nutritional status as it was correlated with dialysis dose. When we extended the observation period of the previous RCT study participants to 10 years [66] and investigated the combination of S-Alb and serum phosphate (S$\mathrm{Pi}$ ) on survival, we found that the best survival was seen in those patients with higher S-Alb and lower S-Pi, while the worst survival was seen in those patients with lower S-Alb and lower S-Pi. In chronic HD patients, S-Pi appears to be a surrogate of protein intake [67].

\section{Weight change during hemodialysis}

During the introductory phase of chronic HD (within 6 months), many patients experience a large weight change; weight decreases in some patients due to mitigation of fluid overload due to edema or heart failure while weight increases in other patients due to recovery of nutritional status. It is important to differentiate between intentional weight loss (gain) in obese (skinny) patients and unintentional change. Chronic excess fluid volume is the main cause of hypertension. In chronic HD patients, seasonal changes in blood pressure and mortality have been report- ed. Blood pressure and mortality increase in winter. Ambient temperature and humidity may change the amount of sweating, physical activity, and other dietary factors. Previously, we observed a seasonal variation in body weight in HD patients living in Okinawa of about $0.5 \mathrm{~kg}$ [68]. This amount of weight change in dialysis patients should not be ignored.

There are multiple causes of cachexia in HD patients including chronic heart failure, stroke, hepatitis-C infection, and malignancy. In particular, malignancy is often detected during the initiation phase [69]. However, among longterm HD patients, these complications are uncommon, conceivably because of the intense medical surveillance these patients are under e.g., visiting medical facilities three times per week. A slight decline in dry weight (DW) could be the first sign of these complications. In addition, depression and cognitive dysfunction are common among HD patients. Loss of appetite is the first sign of these problems. In addition to an adjusted index for malnutrition and inflammation, low functional status is an important predictor of mortality in HD patients [70].

Session time varies with body size and is usually 4 to 6 hours. To comply with these conditions, incident HD patients should be well informed and educated. Salt (sodium) restriction is mandatory for chronic HD patients. To estimate salt intake and intradialytic weight loss, serum sodium levels before and after dialysis are used [71,72]. Estimated median (25th-75th percentile) salt intake was 6.4 g/day (4.6-8.3 g/day) among Japanese HD patients [73]. One-year mortality rate was highest among those with low salt intake ( $<6.0 \mathrm{~g} /$ day). Findings were similar in subgroup analysis based on nutritional parameters such as normalized protein catabolism rate, S-Alb, and BMI. Dong et al. [74] reported that peritoneal dialysis patients with low dietary sodium intake had a higher mortality risk. Their mean (SD) salt intake was lower than Japanese HD patients at $4.6 \mathrm{~g} /$ day (1.9-14.0 g/day). The mechanism underlying these observations remains to be clarified. Other than those complaining of anorexia and those with evidence of PEW, the lower limit of salt intake in CKD patients, including dialysis patients, has not been determined.

\section{Weight change as a predictor of mortality}

Optimal DW is determined using symptoms and hypoten- 
sion during HD sessions as well as parameters that reflect excess fluid volume such as chest X-rays and other laboratory tests. According to the phase 4 study of the Dialysis Outcomes Practice Patterns Study (DOPPS), the practice of deciding DW differs among dialysis facilities and those patients for whom DW is frequently adjusted show better survival [75]. If the DW is achieved, then it means that no excess fluid is present after the HD session. If the amount of fluid removed by HD is too much or the fluid is removed too fast, patients become hypotensive. Post-HD weight is a proxy of DW and refers to the target bodyweight at each HD session; this is usually stable among prevalent HD patients. In patients with a thrice-weekly HD schedule, the first dialysis session of the week may need special attention as the interval from the previous HD is longer at the first session of the week, Monday or Tuesday. Accordingly, weight gain is usually largest at the first HD session of the week. A decline in post-HD weight is a significant predictor of mortality among chronic HD patients.

In a recent study of 461 chronic HD patients with a median follow-up of 10 years, $46 \%$ of patients died [67]. The main causes of death were infection (34\%) and cardiovascular disease (31\%). We showed that in chronic HD patients, risk factors for death differed according to shortterm ( $\leq 3$ years) or long-term ( $>3$ years) observation. Sato et al. [76] showed that the DW obtained from the 1-year observation was a strong predictor of overall death. Kalantar et al. [77] investigated weight changes after initiation of dialysis and found that chronic baseline weight loss had a greater impact on mortality than acute weight loss. However, the optimal speed and amount of weight loss, as well as the optimal duration of observation required, have yet to be determined. Siga et al. [78] used a theoretical approach to determine total mortality in chronic HD patients. Further studies are necessary to define the effects of precisely categorized changes in body weight on survival. It is also important to determine if weight loss or gain is causally associated with mortality.

\section{Current activities in Japan}

Japan and China were known to be high salt intake countries, mainly due to the use of soy sauce and table salt [79]. However, salt intake is gradually decreasing from 2003 to 2019 in Japan according to a national survey conducted by the Ministry of Health, Labor and Welfare (Fig. 5). However, salt intake by men remains higher than that by women. This is likely because men eat out more and drink at bars where they are served finger foods. Alcohol consumption per se is a risk factor for the development [80] and progres-

14

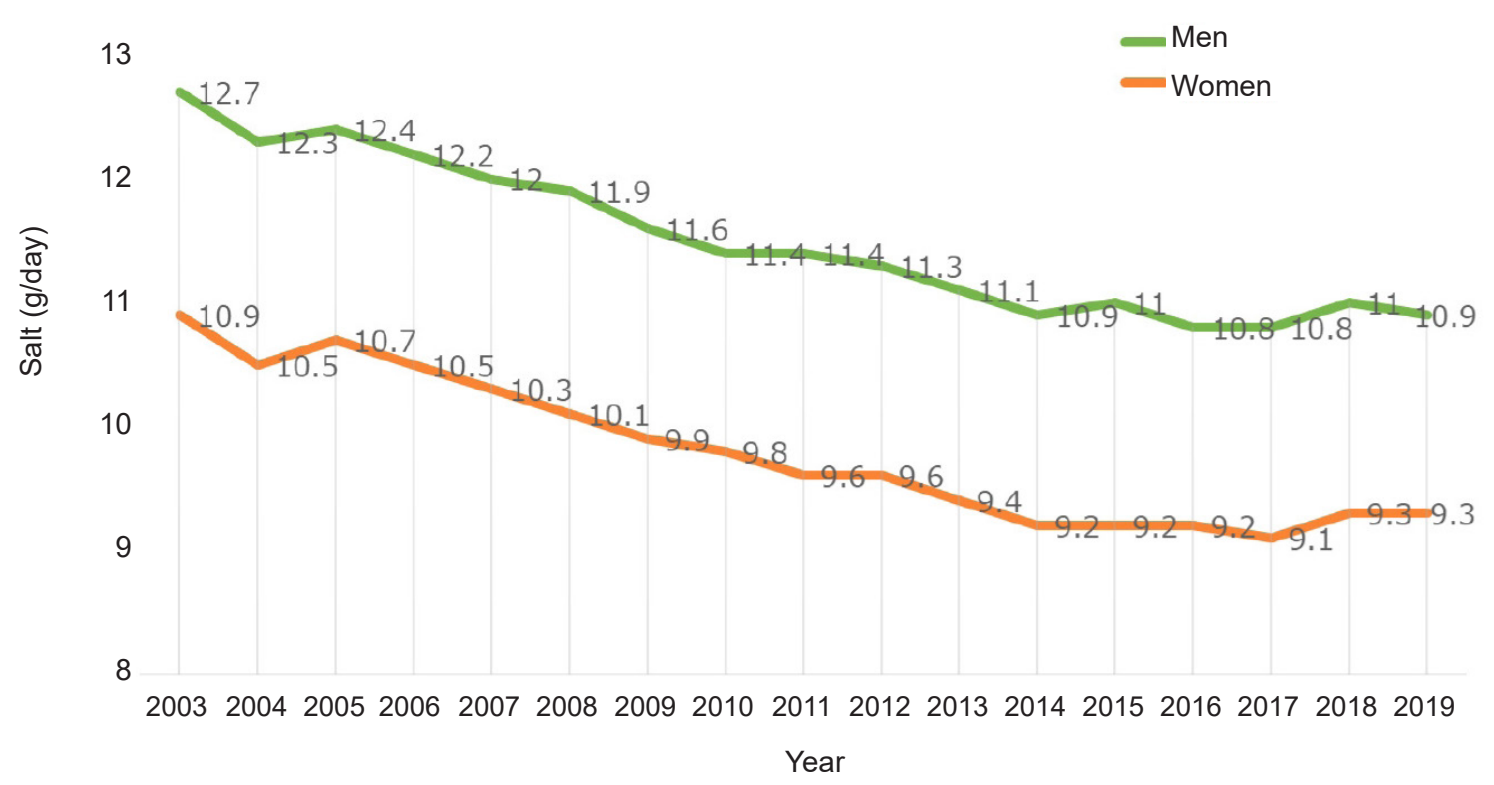

Figure 5. Trends in salt intake in Japan. Data are from the National Survey of the Ministry of Health, Labor and Welfare. 
sion of CKD [81]. Elderly men ( $\geq 60$ years) have a higher intake of salt than younger men. This may be because they have old eating habits and it is more difficult for them to change their dietary habits than younger men. Also, a clear geographic difference in salt intake has been reported in Japan, where it is high in the Hokkaido and Nagano prefectures and lowest in Okinawa. Okinawa is a sub-tropical area; therefore, people do not eat preserved foods such as pickles. Okinawa was occupied after World War II until 1972, therefore the lifestyle is more Americanized than in other areas of Japan. Hokkaido and Nagao are cold in winter, and foods are saltier than in Okinawa. Education on salt restriction is important, in particular in young children $[82,83]$. Recently, school lunch regulations to reduce salt were introduced (https://www.mext.go.jp/a_menu/sports/ syokuiku/1407704.htm). It is hoped that these regulations will promote healthy eating habits in Japan. According to the Ministry of Finance, the amount of salt added to foods by the food processing industry has been decreasing from 2008 to 2018. During these 10 years, the total population has also decreased by about 1.25 million. Nevertheless, salt consumption decreased more than expected. Such a trend could be explained by the efforts of multiple subspecialties other than nephrology such as hypertension [82-84], diabetology, and endocrinology $[85,86]$ societies. In particular, the Japanese Society of Hypertension (JSH) has actively been promoting salt restriction [83]. Details of JSH salt reduction activities have been summarized recently [84]; these activities and target both children and adults, the food industry and food manufacturing companies, as well as the government.

The JSDT annual report stated that the crude annual mortality rate among prevalent HD patients had remained stable at 9\% (1983) and 10\% (2018), despite the large increase in total number of patients from about 53,000 (1983) to 339,000 (2018). Mean age of prevalent dialysis patients increased from 48.3 years old (1983) to 68.8 years old (2018). The percentage of DM increased from $7.4 \%$ (1983) to $39 \%$ (2018) of the prevalent dialysis population. The mean age of incident dialysis patients increased from 51.9 years old

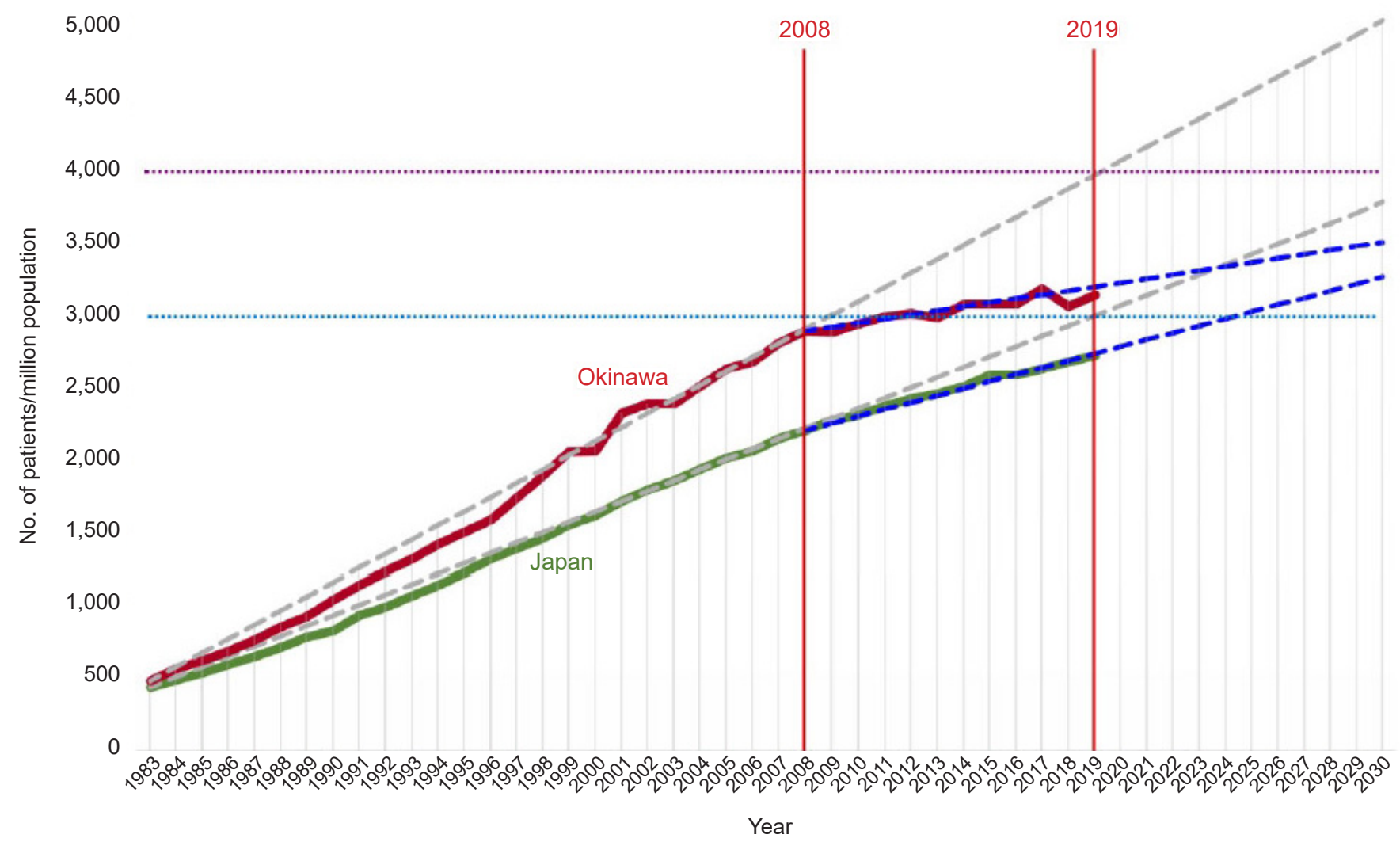

Figure 6. Trends in prevalence, per million population, of chronic dialysis patients in Japan. Data are from the Japanese Society for Dialysis Therapy. Modified and prepared by Iseki K. 
(1983) to 70.0 years old (2018). The incidence of dialysis patients with DM increased from $15.6 \%$ (1983) to $42.3 \%$ (2018). The percentage of chronic glomerulonephritis cases has been declining steadily from $60.0 \%$ in 1983 to $15.6 \%$ in 2018. These trends can be explained by changes in lifestyle, advances in medical treatment, and national health care strategies. Fig. 6 shows trends in the prevalence of dialysis patients in Okinawa and Japan. Until 2008, it increased linearly in both the whole of Japan and Okinawa. Okinawa used to be the prefecture with the highest number of prevalent dialysis patients $[20,87,88]$. However, this appears to have stabilized at around 3,100 per million population.

Renin-angiotensin system blockers are useful for CKD patients with hypertension and proteinuria and have contributed to slowing the progression of CKD to ESKD $[89,90]$. Angiotensin II-receptor blockers (ARBs) have been available in Japan since July 1998. Sodium-glucose cotransporter 2 inhibitors $[91,92]$ to treat DM were introduced in January 2014. The benefits of these drugs are weakened by high salt intake. By contrast, severe salt restriction and/or volume depletion CKD patients may have hyperkalemia. Although we do not have enough information on the use of angiotensin-converting enzyme inhibitor/ARBs in Japanese patients, their use may be higher than in the United States [93]. In addition to these drugs, collaborative efforts among public health nurses and dieticians on dietary management may, at least partly, have contributed to slowing down the rate of increase of dialysis patients. More studies are required to confirm this assumption. Maintaining a healthy lifestyle, including lower salt intake with protein restriction, plays an important role in decreasing the incidence and slowing down the progression of CKD.

\section{Conclusions}

Salt restriction with adequate protein and calorie intake is important in all stages of CKD, and in dialysis patients in particular. Recent reviews and meta-analyses support the benefits of salt reduction [94-96]. Nutritional (salt) management needs to be adjusted for individual lifestyle, dietary habits, and available food types [97], as the elderly population is increasing rapidly in both Japan and Korea. Clinical practice guidelines for CKD patients are mostly based on observational studies and expert opinion. More well-designed observational studies on nutritional man- agement are needed. Too strict salt restriction may decrease quality of life, aggravate $\mathrm{CKD}$, and eventually induce PEW. Sharing knowledge and experience may facilitate observational studies and RCTs to further improve the care of CKD patients [98,99].

\section{Conflicts of interest}

The author has no conflicts of interest to declare.

\section{Acknowledgments}

I would like to thank the organizing committee of the KSN2021 (Professor Won Kim, the Congress President; Professor Chul Woo Yang, President of the Korean Society of Nephrology; and Professor Sang-Ho Lee, the Chair Scientific Committee) and Professor Tae-Hyun Yoo MD, Ph.D., the Editor-in-Chief of Kidney Research and Clinical Practice.

\section{ORCID}

Kunitoshi Iseki, https://orcid.org/0000-0001-6624-0216

\section{References}

1. Jha V, Garcia-Garcia G, Iseki K, et al. Chronic kidney disease: global dimension and perspectives. Lancet 2013;382:260-272.

2. Park S, Lee S, Kim Y, et al. Reduced risk for chronic kidney disease after recovery from metabolic syndrome: a nationwide population-based study. Kidney Res Clin Pract 2020;39:180-191.

3. Ikizler TA, Burrowes JD, Byham-Gray LD, et al. KDOQI clinical practice guideline for nutrition in CKD: 2020 update. Am J Kidney Dis 2020;76(3 Suppl 1):S1-S107.

4. Clinical practice guidelines for nutrition in chronic renal failure. K/DOQI, National Kidney Foundation. Am J Kidney Dis 2000; 35(6 Suppl 2):S17-S104.

5. Kidney Disease: Improving Global Outcomes (KDIGO) CKD Work Group. KDIGO 2012 clinical practice guideline for the evaluation and management of chronic kidney disease. Kidney Int Suppl 2013;3:1-150.

6. Japanese Society of Nephrology. Evidence-based clinical practice guideline for CKD 2018 [Internet]. Tokyo: Tokyo Iagakusha; 2018 [cited 2021 Sep 1]. Available from: https://cdn.jsn.or.jp/ data/guideline-20180604.pdf. 
7. Sasaki S. Preface: evidence-based practice guideline for the treatment of chronic kidney disease. Clin Exp Nephrol 2009; 13:533.

8. Japanese Society of Nephrology. Essential points from evidence-based clinical practice guidelines for chronic kidney disease 2018. Clin Exp Nephrol 2019;23:1-15.

9. Shemin D, Dworkin LD. Sodium balance in renal failure. Curr Opin Nephrol Hypertens 1997;6:128-132.

10. Kidney Disease: Improving Global Outcomes (KDIGO) Blood Pressure Work Group. KDIGO 2021 clinical practice guideline for the management of blood pressure in chronic kidney disease. Kidney Int 2021;99(3S):S1-S87.

11. Kistler BM, Moore LW, Benner D, et al. The International Society of Renal Nutrition and Metabolism commentary on the National Kidney Foundation and Academy of Nutrition and Dietetics KDOQI clinical practice guideline for nutrition in chronic kidney disease. J Ren Nutr 2021;31:116-120.

12. Kistler BM, Benner D, Burrowes JD, et al. Eating during hemodialysis treatment: a consensus statement from the International Society of Renal Nutrition and Metabolism. J Ren Nutr 2018;28:4-12.

13. Iseki K, Yamagata K. A practical approach of salt and protein restriction for CKD patients in Japan. BMC Nephrol 2016;17:87.

14. Wakasugi M, James Kazama J, Narita I. Associations between the intake of miso soup and Japanese pickles and the estimated 24hour urinary sodium excretion: a population-based cross-sectional study. Intern Med 2015;54:903-910.

15. Yazaki Y, Kadowaki T. Combating diabetes and obesity in Japan. Nat Med 2006;12:73-74.

16. Alberti KG, Eckel RH, Grundy SM, et al. Harmonizing the metabolic syndrome: a joint interim statement of the International Diabetes Federation Task Force on Epidemiology and Prevention; National Heart, Lung, and Blood Institute; American Heart Association; World Heart Federation; International Atherosclerosis Society; and International Association for the Study of Obesity. Circulation 2009;120:1640-1645.

17. Kohro T, Furui Y, Mitsutake N, et al. The Japanese national health screening and intervention program aimed at preventing worsening of the metabolic syndrome. Int Heart J 2008;49:193-203.

18. Ninomiya T, Kiyohara Y, Kubo M, et al. Metabolic syndrome and CKD in a general Japanese population: the Hisayama Study. Am J Kidney Dis 2006;48:383-391.

19. Tozawa M, Iseki C, Tokashiki K, et al. Metabolic syndrome and risk of developing chronic kidney disease in Japanese adults. Hypertens Res 2007;30:937-943.
20. Iseki K. Metabolic syndrome and chronic kidney disease: a Japanese perspective on a worldwide problem. J Nephrol 2008;21: 305-312.

21. Yamagata K, Ishida K, Sairenchi T, et al. Risk factors for chronic kidney disease in a community-based population: a 10-year follow-up study. Kidney Int 2007;71:159-166.

22. Ohta Y, Tsuchihashi T, Kiyohara K, Oniki H. High salt intake promotes a decline in renal function in hypertensive patients: a 10year observational study. Hypertens Res 2013;36:172-176.

23. Sugiura T, Takase H, Ohte N, Dohi Y. Dietary salt intake is a significant determinant of impaired kidney function in the general population. Kidney Blood Press Res 2018;43:1245-1254.

24. Kelly JT, Su G, Zhang L, et al. Modifiable lifestyle factors for primary prevention of CKD: a systematic review and meta-analysis. J Am Soc Nephrol 2021;32:239-253.

25. Yano Y, Fujimoto S, Sato Y, et al. Association between prehypertension and chronic kidney disease in the Japanese general population. Kidney Int 2012;81:293-299.

26. Iseki K, Asahi K, Moriyama T, et al. Risk factor profiles based on estimated glomerular filtration rate and dipstick proteinuria among participants of the Specific Health Check and Guidance System in Japan 2008. Clin Exp Nephrol 2012;16:244-249.

27. Iseki K, Konta T, Asahi K, et al. Dipstick proteinuria and all-cause mortality among the general population. Clin Exp Nephrol 2018; 22:1331-1340.

28. Wakasugi M, Kazama J, Narita I, et al. Association between overall lifestyle changes and the incidence of proteinuria: a population-based, cohort study. Intern Med 2017;56:1475-1484.

29. Kudo A, Asahi K, Satoh H, et al. Fast eating is a strong risk factor for new-onset diabetes among the Japanese general population. Sci Rep 2019;9:8210.

30. Yoon CY, Noh J, Lee J, et al. High and low sodium intakes are associated with incident chronic kidney disease in patients with normal renal function and hypertension. Kidney Int 2018;93: 921-931.

31. DiNicolantonio JJ, Niazi AK, Sadaf R, O’ Keefe JH, Lucan SC, Lavie CJ. Dietary sodium restriction: take it with a grain of salt. Am J Med 2013;126:951-955.

32. Ritz E, Schwenger V. Lifestyle modification and progressive renal failure. Nephrology (Carlton) 2005;10:387-392.

33. de Francisco AL, Fresnedo GF, Palomar R, Piñera C, Arias M. The renal benefits of a healthy lifestyle. Kidney Int Suppl 2005;99:S2S6.

34. Iseki K, Tohyama K, Matsumoto T, Nakamura H. High prevalence of chronic kidney disease among patients with sleep relat- 
ed breathing disorder (SRBD). Hypertens Res 2008;31:249-255.

35. Sakaguchi Y, Shoji T, Kawabata H, et al. High prevalence of obstructive sleep apnea and its association with renal function among nondialysis chronic kidney disease patients in Japan: a cross-sectional study. Clin J Am Soc Nephrol 2011;6:995-1000.

36. Sakaguchi Y, Hatta T, Hayashi T, et al. Association of nocturnal hypoxemia with progression of CKD. Clin J Am Soc Nephrol 2013; 8:1502-1507.

37. Nakamura K, Nakamura H, Tohyama K, et al. Survival benefit of continuous positive airway pressure in Japanese patients with obstructive sleep apnea: a propensity-score matching analysis. $J$ Clin Sleep Med 2021;17:211-218.

38. Iseki K; for the Okinawa Dialysis Study (OKIDS) Group. Analysis of referral pattern and survival in chronic dialysis patients in Okinawa, Japan (1993-1997). Clin Exp Nephrol 2002;6:43-48.

39. Sprangers B, Evenepoel P, Vanrenterghem Y. Late referral of patients with chronic kidney disease: no time to waste. Mayo Clin Proc 2006;81:1487-1494.

40. Campbell GA, Bolton WK. Referral and comanagement of the patient with CKD. Adv Chronic Kidney Dis 2011;18:420-427.

41. Rysz J, Franczyk B, Ławiński J, Olszewski R, Ciałkowska-Rysz A, Gluba-Brzózka A. The impact of CKD on uremic toxins and gut microbiota. Toxins (Basel) 2021;13:252.

42. Sumida K, Kovesdy CP. The gut-kidney-heart axis in chronic kidney disease. Physiol Int 2019;106:195-206.

43. Sumida K, Molnar MZ, Potukuchi PK, et al. Constipation and Incident CKD. J Am Soc Nephrol 2017;28:1248-1258.

44. Sofue T, Okano Y, Matsushita N, et al. The effects of a participatory structured group educational program on the development of CKD: a population-based study. Clin Exp Nephrol 2019;23:10311038.

45. Niu SF, Wu CK, Chuang NC, Yang YB, Chang TH. Early Chronic Kidney Disease Care Programme delays kidney function deterioration in patients with stage I-IIIa chronic kidney disease: an observational cohort study in Taiwan. BMJ Open 2021;11:e041210.

46. Oellgaard J, Gæde P, Rossing P, Persson F, Parving HH, Pedersen O. Intensified multifactorial intervention in type 2 diabetics with microalbuminuria leads to long-term renal benefits. Kidney Int 2017;91:982-988.

47. Navaneethan SD, Zoungas S, Caramori ML, et al. Diabetes management in chronic kidney disease: synopsis of the 2020 KDIGO Clinical Practice Guideline. Ann Intern Med 2021;174:385-394.

48. Yamagata K, Makino H, Iseki K, et al. Effect of behavior modification on outcome in early- to moderate-stage chronic kidney disease: a cluster-randomized trial. PLoS One 2016;11:e0151422.

49. Hoshino J, Nagai K, Kai H, et al. A nationwide prospective cohort study of patients with advanced chronic kidney disease in Japan: the Reach-J CKD cohort study. Clin Exp Nephrol 2018;22:309317.

50. Okubo R, Kondo M, Hoshi SL, et al. Cost-effectiveness of behavior modification intervention for patients with chronic kidney disease in the FROM-J Study. J Ren Nutr 2021;31:484-493.

51. McMahon EJ, Campbell KL, Bauer JD. Taste perception in kidney disease and relationship to dietary sodium intake. Appetite 2014;83:236-241.

52. Kang M, Kang E, Ryu H, et al. Measured sodium excretion is associated with CKD progression: results from the KNOW-CKD study. Nephrol Dial Transplant 2021;36:512-519.

53. Garofalo C, Provenzano M, Andreucci M, et al. Predictive effect of salt intake on patient and kidney survival in non-dialysis CKD: competing risk analysis in older versus younger patients under nephrology care. Nephrol Dial Transplant 2021;36:22322240.

54. Himmelfarb J, Vanholder R, Mehrotra R, Tonelli M. The current and future landscape of dialysis. Nat Rev Nephrol 2020;16:573585.

55. Eknoyan G, Beck GJ, Cheung AK, et al. Effect of dialysis dose and membrane flux in maintenance hemodialysis. $N$ Engl J Med 2002;347:2010-2019.

56. Twardowski ZJ, Misra M, Singh AK. Con: Randomized controlled trials (RCT) have failed in the study of dialysis methods. Nephrol Dial Transplant 2013;28:826-832.

57. Singh AK, Kari JA, Twardowski ZJ, Misra M. Pro: The value of randomized controlled studies in dialysis methods. Nephrol Dial Transplant 2013;28:834-836.

58. Van Biesen W, Lameire N. Moderator's view: Are alternative dialysis strategies superior to conventional dialysis: what Popper tells us. Nephrol Dial Transplant 2013;28:837-838.

59. Goodkin DA, Young EW, Kurokawa K, Prütz KG, Levin NW. Mortality among hemodialysis patients in Europe, Japan, and the United States: case-mix effects. Am J Kidney Dis 2004;44(5 Suppl 2):16-21.

60. Kalantar-Zadeh K, Wightman A, Liao S. Ensuring choice for people with kidney failure: dialysis, supportive care, and hope. $N$ Engl J Med 2020;383:99.

61. Fernández-Martín JL, Martínez-Camblor P, Dionisi MP, et al. Improvement of mineral and bone metabolism markers is associated with better survival in haemodialysis patients: the COSMOS study. Nephrol Dial Transplant 2015;30:1542-1551. 
62. Fouque D, Kalantar-Zadeh K, Kopple J, et al. A proposed nomenclature and diagnostic criteria for protein-energy wasting in acute and chronic kidney disease. Kidney Int 2008;73:391-398.

63. Kalantar-Zadeh K, Fouque D. Nutritional management of chronic kidney disease. N Engl J Med 2017;377:1765-1776.

64. Koppe L, Fouque D, Kalantar-Zadeh K. Kidney cachexia or protein-energy wasting in chronic kidney disease: facts and numbers. J Cachexia Sarcopenia Muscle 2019;10:479-484.

65. Iseki K, Kawazoe N, Fukiyama K. Serum albumin is a strong predictor of death in chronic dialysis patients. Kidney Int 1993;44: 115-119.

66. Iseki K, Arima H, Kohagura K, et al. Effects of angiotensin receptor blockade (ARB) on mortality and cardiovascular outcomes in patients with long-term haemodialysis: a randomized controlled trial. Nephrol Dial Transplant 2013;28:1579-1589.

67. Moromizato T, Kohagura K, Tokuyama K, et al. Predictors of survival in chronic hemodialysis patients: a 10-year longitudinal follow-up analysis. Am J Nephrol 2021;52:108-118.

68. Tozawa M, Iseki K, Iseki C, Morita O, Yoshi S, Fukiyama K. Seasonal blood pressure and body weight variation in patients on chronic hemodialysis. Am J Nephrol 1999;19:660-667.

69. Iseki K, Osawa A, Fukiyama K. Evidence for increased cancer deaths in chronic dialysis patients. Am J Kidney Dis 1993;22:308313.

70. Kanda E, Lopes MB, Tsuruya K, et al. The combination of malnutrition-inflammation and functional status limitations is associated with mortality in hemodialysis patients. Sci Rep 2021;11:1582.

71. Kimura G, Kojima S, Saito F, et al. Quantitative estimation of dietary intake in patients on hemodialysis. Int J Artif Organs 1988;11:161-168.

72. Ramdeen G, Tzamaloukas AH, Malhotra D, Leger A, Murata GH. Estimates of interdialytic sodium and water intake based on the balance principle: differences between nondiabetic and diabetic subjects on hemodialysis. ASAIO J 1998;44:812-817.

73. Ikenoue T, Koike K, Fukuma S, Ogata S, Iseki K, Fukuhara S. Salt intake and all-cause mortality in hemodialysis patients. Am J Nephrol 2018;48:87-95.

74. Dong J, Li Y, Yang Z, Luo J. Low dietary sodium intake increases the death risk in peritoneal dialysis. Clin J Am Soc Nephrol 2010;5:240-247.

75. Dasgupta I, Thomas GN, Clarke J, et al. Associations between hemodialysis facility practices to manage fluid volume and intradialytic hypotension and patient outcomes. Clin J Am Soc Nephrol 2019;14:385-393.
76. Sato Y, Toida T, Nakagawa H, et al. Diminishing dry weight is strongly associated with all-cause mortality among longterm maintenance prevalent dialysis patients. PLoS One 2018;13:e0203060.

77. Kalantar-Zadeh K, Streja E, Molnar MZ, et al. Mortality prediction by surrogates of body composition: an examination of the obesity paradox in hemodialysis patients using composite ranking score analysis. Am J Epidemiol 2012;175:793-803.

78. Siga MM, Ducher M, Florens N, et al. Prediction of all-cause mortality in haemodialysis patients using a Bayesian network. Nephrol Dial Transplant 2020;35:1420-1425.

79. Brown IJ, Tzoulaki I, Candeias V, Elliott P. Salt intakes around the world: implications for public health. Int J Epidemiol 2009; 38:791-813.

80. Kimura Y, Yamamoto R, Shinzawa M, et al. Alcohol consumption and incidence of proteinuria: a retrospective cohort study. Clin Exp Nephrol 2018;22:1133-1142.

81. Joo YS, Koh H, Nam KH, et al. Alcohol consumption and progression of chronic kidney disease: results from the Korean Cohort Study for Outcome in Patients with Chronic Kidney Disease. Mayo Clin Proc 2020;95:293-305.

82. Yasutake K, Nagafuchi M, Tanaka T, et al. Necessity of salt intake reduction education beginning in youth: a cross-sectional survey of sodium-to-potassium ratios in mothers and their preschool children. Hypertens Res 2021;44:1307-1315.

83. Tsuchihashi T, Ishimitsu T, Ando K, et al. JSH Statement: Tokyo declaration promoting salt reduction by the Japanese Society of Hypertension-the JSH Tokyo declaration. Hypertens Res 2020; 43:1133-1134.

84. Tsuchihashi T. Practical and personal education of dietary therapy in hypertensive patients. Hypertens Res 2020;43:6-12.

85. Ueki K, Sasako T, Kato M, et al. Design of and rationale for the Japan Diabetes Optimal Integrated Treatment study for 3 major risk factors of cardiovascular diseases (J-DOIT3): a multicenter, open-label, randomized, parallel-group trial. BMJ Open Diabetes Res Care 2016;4:e00123.

86. Ueki K, Sasako T, Okazaki Y, et al. Effect of an intensified multifactorial intervention on cardiovascular outcomes and mortality in type 2 diabetes (J-DOIT3): an open-label, randomised controlled trial. Lancet Diabetes Endocrinol 2017;5:951-964.

87. Iseki K, Kohagura K, Sakima A, et al. Changes in the demographics and prevalence of chronic kidney disease in Okinawa, Japan (1993 to 2003). Hypertens Res 2007;30:55-62.

88. Usami T, Koyama K, Takeuchi O, Morozumi K, Kimura G. Regional variations in the incidence of end-stage renal failure in 
Japan. JAMA 2000;284:2622-2624.

89. Silvariño R, Rios P, Baldovinos G, et al. Is chronic kidney disease progression influenced by the type of renin-angiotensin-system blocker used? Nephron 2019;143:100-107.

90. McCoy IE, Han J, Montez-Rath ME, Chertow GM. Barriers to ACEI/ARB use in proteinuric chronic kidney disease: an observational study. Mayo Clin Proc 2021;96:2114-2122.

91. Zinman B, Wanner C, Lachin JM, et al. Empagliflozin, cardiovascular outcomes, and mortality in type 2 diabetes. $N$ Engl J Med 2015;373:2117-2128.

92. Heerspink HJ, Stefánsson BV, Correa-Rotter R, et al. Dapagliflozin in patients with chronic kidney disease. $N$ Engl J Med 2020;383:1436-1446.

93. Foti KE, Wang D, Chang AR, et al. Potential implications of the 2021 KDIGO blood pressure guideline for adults with chronic kidney disease in the United States. Kidney Int 2021;99:686-695.
94. McMahon EJ, Campbell KL, Bauer JD, Mudge DW, Kelly JT. Altered dietary salt intake for people with chronic kidney disease. Cochrane Database Syst Rev 2021;6:CD010070.

95. Jain N, Reilly RF. Effects of dietary interventions on incidence and progression of CKD. Nat Rev Nephrol 2014;10:712-724.

96. Garofalo C, Borrelli S, Provenzano M, et al. Dietary salt restriction in chronic kidney disease: a meta-analysis of randomized clinical trials. Nutrients 2018;10:732.

97. Wang AY, Kalantar-Zadeh K, Fouque D, et al. Precision medicine for nutritional management in end-stage kidney disease and transition to dialysis. Semin Nephrol 2018;38:383-396.

98. Kim SM, Jung JY. Nutritional management in patients with chronic kidney disease. Korean J Intern Med 2020;35:1279-1290.

99. Kim Y, Park CW. Can management of the components of metabolic syndrome modify the course of chronic kidney disease? Kidney Res Clin Pract 2020;39:118-120. 